Abstract P2-S5.05 Table 1. 4 Latent class model of Winnipeg street population risk profiles

\begin{tabular}{|c|c|c|c|c|}
\hline Class r & ities: Gamma est & & & \\
\hline Class & 1 & 2 & 3 & 4 \\
\hline & $0.2474(0.0254)$ & $0.3099(0.0315)$ & $0.2137(0.0243)$ & $0.2289(0.0325)$ \\
\hline
\end{tabular}

Item response probabilities: $\rho$ estimates (SEs)

\begin{tabular}{|c|c|c|c|c|c|}
\hline HSA14_: & $0.0335(0.0482)$ & $0.7211(0.0372)$ & $0.6719(0.0473)$ & $0.7207(0.0467)$ & Mental health (even been $\mathrm{dx}$ ) \\
\hline ALC2_: & $0.3754(0.0461)$ & $0.8494(0.0627)$ & $0.4599(0.0505)$ & $0.0623(0.0433)$ & Binge drinking ( $>5$ drinks) \\
\hline CRS1_: & $0.3158(0.0540)$ & $0.9773(0.0166)$ & $0.9128(0.0342)$ & $0.9258(0.0315)$ & Crack use \\
\hline SSU1_: & $0.1425(0.0349)$ & $0.5256(0.0414)$ & $0.5068(0.0525)$ & $0.5541(0.0516)$ & Solvent use \\
\hline idu1_: & $0.0488(0.0285)$ & $0.6211(0.0404)$ & $0.6698(0.0568)$ & $0.6879(0.0525)$ & Injection drug use \\
\hline SNSX7_new_: & $0.1944(0.0403)$ & $0.7745(0.0368)$ & $0.0052(0.0069)$ & $0.6961(0.0511)$ & Sex partners have multiple part \\
\hline SNID1_: & $0.1784(0.0398)$ & $0.5803(0.0407)$ & $0.3842(0.0483)$ & $0.7888(0.0477)$ & Network members' IDU \\
\hline SNALC_: & $0.6019(0.0459)$ & $0.9870(0.0158)$ & $0.4698(0.0500)$ & $0.4151(0.0713)$ & Network members' alcohol use \\
\hline SNCRACK_: & $0.0136(0.0137)$ & $0.6265(0.0408)$ & $0.3629(0.0498)$ & $0.4515(0.0549)$ & Network members' crack use \\
\hline SNODU_: & $0.4989(0.0462)$ & $0.8380(0.0331)$ & $0.4820(0.0497)$ & $0.5634(0.0518)$ & Network members' other drug use \\
\hline SNSX1_: & $0.5883(0.0496)$ & $0.9872(0.0121)$ & $0.1129(0.0572)$ & $0.9958(0.0068)$ & Network members are sex part. \\
\hline
\end{tabular}

health issues as well as individual crack, solvent use, and injection use but no network level correlates while the fourth latent class (23\%) was distinguished for engaging in risky sexual behaviours and having these risky behaviours be supported at the social network level.

Conclusions Latent class analysis demonstrated that there are indeed subgroups of vulnerable populations who warrant targeted interventions given their different risk profiles. This type of investigation offers a public health population segmentation strategy to plan for future targeted prevention efforts that can more effectively address the special needs of these subgroups of vulnerable populations.

\section{P2-S5.06 BEHAVIOURAL INTERVENTIONS FOR REDUCING HIV INFECTION IN WORKERS IN OCCUPATIONAL SETTINGS, A COCHRANE SYSTEMATIC REVIEW}

doi:10.1136/sextrans-2011-050108.348

${ }^{1} \mathrm{O}$ Ojo, ${ }^{2} \mathrm{~J}$ Verbeek, ${ }^{1} \mathrm{~K}$ Rasanen, ${ }^{1} \mathrm{~J}$ Heikkinen, ${ }^{3} \mathrm{~L}$ Isotalo, ${ }^{4} \mathrm{~N}$ Mngoma, ${ }^{5} \mathrm{E}$ Ruotsaalainen. ${ }^{1}$ University of Eastern Finland, Kuopio, Finland; ${ }^{2}$ Finnish Institute of Occupational health, Cochrane Occupational safety and health group, Kuopio, Finland; ${ }^{3}$ Finnish Institute of Occupational Health, Helsinki, Finland; ${ }^{4}$ Queen's University, Kingston, Canada; ${ }^{5}$ Kuopio University Hospital, Kuopio, Finland

Background HIV in the workforce has a negative impact on economic growth and development of affected countries. The workplace provides an important avenue to prevent HIV by providing easy access to workers who might have similar high risk sexual behaviour because of the nature of their work. Objective of this systematic review is to evaluate the effect of behavioural interventions on high risk sexual behaviour to prevent HIV among workers.

Methods We searched electronic databases to locate studies. Only randomised control studies in occupational settings or among workers identified as high risk groups were included. The outcome measures were: indicators of high risk sexual behaviours, uptake of Voluntary Counselling and Testing (VCT), and incident cases of HIV. We excluded health workers, sex workers, injection drug users and Men who have sex with men because these are already in other reviews.

Results We included 4 studies but we had incomplete data from one. All the studies were from developing countries in Asia and Africa with a total of 7994 participants. We grouped the studies in the following comparisons: Uptake of VCT was increased when provided in workplace compared to voucher for testing only, $\mathrm{RR}=14.0$ (95\% CI 11.8 to 16.7), HIV incidence was not reduced, $\mathrm{RR}=1.4$ (95\% CI 0.7 to 2.7$)$. Education caused a non-significant decrease in sex with extra partner $\mathrm{RR}=0.7$ (95\% CI 0.3 to 1.8$)$ and unprotected sex, $\mathrm{RR}=0.9$ ( $95 \% \mathrm{CI} 0.5$ to 1.4 ). More intensive Information, motivation and behavioural (IMB) skills training increased the use of condom, $\mathrm{RR}=2.8$ (95\% CI 1.6 to 4.1$)$ and decreased sex with commercial sex workers, $R R=0.8$ (95\% CI 0.80 to $0.96)$ in a very high risk group of truck drivers.

Conclusion VCT might not be effective in reducing HIV incidence in workplace intervention, and delivering voluntary counselling and testing on-site at workplace increases the uptake of VCT. However, education, and Information, motivation and behavioural (IMB) skills training might be worthwhile in reducing risky sexual behaviour and increasing condom use, especially when delivered with high intensity in the workplace.

\section{P2-S5.07 COST OF THE AVAHAN HIV PREVENTION PROGRAMME FOR HIGH RISK GROUPS: RESULTS FROM 23 DISTRICTS FROM FOUR SOUTHERN STATES IN INDIA}

doi:10.1136/sextrans-2011-050108.349

${ }^{1,2} \mathrm{~S}$ Chandrashekar, ${ }^{1} \mathrm{~A}$ Vassall, ${ }^{3} \mathrm{~B}$ Reddy, ${ }^{3} \mathrm{G}$ Shetty, ${ }^{4} \mathrm{M}$ Alary, ${ }^{3} \mathrm{P}$ Vickerman. ${ }^{1}$ London School of Hygiene and Tropical Medicine, London, UK; ${ }^{2}$ St. John's Research Institute, SJRI Bangalore, India; ${ }^{3}$ Karnataka Health Promotion trust, Bangalore, India; ${ }^{4}$ Centre hospitalier affilié universitaire de Québec, Quebec, Canada

Background It is estimated that 2.27 million people are living with HIV or AIDS in India. The epidemic is concentrated and predominately driven by marginalised groups. The Avahan Programme in India is one of the largest HIV prevention programmes targeted at high risk populations within a single country in the world. It provides grants to state lead partners (SLPs), who in turn provide grants to non-government organsiations (NGOs) at the district level to deliver multi-component interventions (including peer outreach, STI services, and structural interventions). This study presents the costs of implementing these interventions to female sex workers, men who have sex with men and transgender from 23 districts in the four Southern states of India over 4 years.

Methods Financial and economic costs were prospectively collected. Costs by input and activity, unit costs of interventions between 2004 and 2008 were analysed. Economic costs are presented in US \$ 2008 using $3 \%$ discount rate. 
Results The total economic cost of the intervention over 4 years for 23 districts was US\$27341121(range per district US\$307597-US $\$ 3147790$ ) for an estimated target population of 93345. In year 4, the average annual economic cost per estimated population at the NGO level across the 23 districts was US\$70 (US\$30-174). The average annual financial cost at current prices was US\$59. Over the four years, at the NGO level, capital costs accounted for $10 \%$ of total costs. The main recurrent costs were personnel costs (46\%) and the materials and supplies for sexually transmitted infections (STI) services (13\%). Examining both SLP and NGO costs, programme management, information and grant management costs activities accounted for $27 \%$ of total cost; followed by capacity building $(20 \%)$, STI services (19\%), peer outreach (including behaviour change communication, condom provision) (16\%) and structural interventions (11\%). The proportion of cost that was spent on direct services such as peer outreach and STI services increased as the programme scaled up. Costs for structural activities also increased from $5 \%$ in year 1 to $11 \%$ by year 4 .

Conclusions Assessing costs over the life of the project helps to identify how costs vary with the changing needs and strategies of the programme. We will conduct further analysis to examine which factors most influence costs (local price/wages, programme intensity, community involvement etc). This cost data can assist the realistic planning of large scale long term HIV prevention programmes in the future.

\section{P2-S5.08 KNOWLEDGE ABOUT STD TRANSMISSION AND CLINICAL CHARACTERISTICS AMONG CONSCRIPTS IN BRAZIL, 2007}

doi:10.1136/sextrans-2011-050108.350

${ }^{1} \mathrm{D}$ Ribeiro, ${ }^{1} \mathrm{~V}$ M Pinto, ${ }^{2} \mathrm{~A}$ Espinosa, ${ }^{1} \mathrm{E}$ Rezende, ${ }^{1} \mathrm{G}$ Pereira, ${ }^{3} \mathrm{M}$ Barbosa. ${ }^{1}$ Ministry of Health, Brasilia, Brazil; ${ }^{2}$ UFES, Brazil; ${ }^{3}$ Federal District Government, Brazil

Our goal was to describe knowledge of military conscripts related to STDs. A self-applied questionnaire with demographic characteristics and STD clinics; knowledge of transmission modes and preferred access to information about STDs was applied. Variables associated with outcome were: being 19-20 years [OR 1.2 (95\% CI 1.18 to 1.32)]; STD transmission by eating contaminated food [OR 2.2 (95\% CI 1.96 to 2.55)], for bathing in rivers / beaches [OR $1.5(95 \%$ CI 1.27 to 1.88 )]; by mosquitoes [OR 1.5 (95\% CI 1.38 to 1.65$)$ ], and for having sexual intercourse $\leq 14$ years [OR 1.4 (95\% CI 1.33 to $1.55)]$. The variables were negatively associated: being white [OR 0.9 (95\% CI 0.82 to 0.91$)$ ], namely the transmission by sharing syringes and needles [OR 0.7 (95\% CI 0.62 - to 0.78 )], by transmission from mother to child during childbirth and breastfeeding [OR 0.6 (95\% CI 0.58 to 0.69 )], using a condom at last sex [OR 0.8 (95\% CI 0.71 to 0.85$)$ ]; MSM [OR 0.7 (95\% CI 0.60 to 0.92 ) and know that sex without condom use increases the risk of transmission [OR 0, 4 (95\% CI 0.37 to 0.51)]. STD education programs must be dynamic and comprehensive, involving teens, their parents and educators, once isolated knowledge about modes of transmission of STD is not enough to help protect.

\section{P2-S5.09 TRANSGENDERS AND HIV: A LITERATURE REVIEW OF HIV RISK BEHAVIOURS AND PREVALENCE RATES}

doi:10.1136/sextrans-2011-050108.351

${ }^{1} \mathrm{~S}$ Butt, ${ }^{1} \mathrm{~L}$ Mena, ${ }^{2} \mathrm{C}$ Muzny. ${ }^{1}$ University of Mississippi Medical Center, Jackson, USA; ${ }^{2}$ University of Alabama, Birmingham, USA

Background Transgenders (TG) are a marginalised population that has been greatly impacted by the HIV/AIDS epidemic. The objective of this study is to review the current literature citing risk factors and prevalence rates for HIV infection among male to female (MTF) and female to male (FTM) TG in the USA.

Methods A review of the original research articles published from 1981 to 2010 was performed through a Pubmed search using the terms "HIV risk" and "Transgender".

Results Thirty-five articles were identified, with MTF described in thirty-three and FTM described in nine. Collectively all racial groups were represented. The majority of research was done in large urban cities in the Southwest, Midwest, and Northeast. Most studies relied on self-report of HIV infection; and only six studies tested participants for HIV. The prevalence rates of HIV infection ranged from $2.2 \%$ to $68.0 \%$. Unprotected receptive anal intercourse (URAI), commercial sex work (CSW), and African American (AA) race were the most frequent HIV risk factors identified. Homelessness and financial instability were frequent among adolescents. When compared to men who have sex with men (MSM) and heterosexual females, MTF are more likely to have URAI with multiple sexual partners. Among MTF participants, AA and Hispanics were more likely to participate in CSW than Caucasians. Self reported prevalence of HIV was low in FTM population (0-2\%), except in one study which tested for HIV and found similar HIV prevalence in both FTM (10\%) and MTF (10\%); risk factors associated with HIV infection in this study were unprotected sex with a partner of unknown HIV status and location in an urban metropolitan area. Conclusions HIV rates among TG are high, especially among AA and Hispanics. Majority of MTF participate in high risk sexual activities. Geographically and ethnically diverse studies including MTF and FTM are needed to test HIV prevalence and associated risk factors in TG population.

\section{P2-S5.10 SEXUAL BEHAVIOURS AMONG INJECTING DRUG USERS (IDUS) IN NORTH-EAST INDIA}

doi:10.1136/sextrans-2011-050108.352

\section{R K Mishra. Emmanuel Hospital Association, Guwahati, India}

Background The North-East Indian states of Nagaland and Manipur have among the highest HIV prevalence in India, fuelled by an epidemic of injecting drug use. In 2008, the HIV prevalence among IDUs in Manipur was 28.65\% and in Nagaland was 3.16\% in 2008 as per National Surveillance data. Proximity to Myanmar, high unemployment and ongoing violent insurgencies serve to exacerbate the problem. Project ORCHID, an initiative funded by the Bill and Melinda Gates Foundation, has been running HIV prevention programs with high risk groups through local NGOs in these two states since 2004. Methodology: To evaluate risk behaviours among the IDU communities served by Project ORCHID, polling booth surveys (PBS) were conducted in all 26 intervention sites across both states in 2010. Participants were purposively selected from a broad geographical area, and asked to anonymously answer questions through a ballot box.

Results In total, 558 IDUs in Manipur and 440 IDUs in Nagaland were sampled for the PBS. They reported high rates of access to needles/syringes from ORCHID projects (82\% in Manipur and 96\% in Nagaland). In both states, three-quarters had injected in the previous week. Among these, the mean weekly injection frequency was 11 times in Manipur and 5 in Nagaland. The proportion of injecting episodes using a clean needle/syringe was $77 \%$ in Manipur and $94 \%$ in Nagaland. Needle sharing in the previous month was reported by 38\% in Manipur and 25\% in Nagaland. Manipur had less IDUs who had been sexually active in the previous week $(46 \%$ vs $71 \%$ in Nagaland) but consistent condom use was lower in Manipur than Nagaland (30\% vs $76 \%$ ).

Conclusion Higher rates of risky injecting and sexual behaviour were found in Manipur IDUs, which together with the much higher 